\title{
REVISTAACIÓN
}

Revista Educación

ISSN: 0379-7082

ISSN: 2215-2644

revedu@gmail.com

Universidad de Costa Rica

Costa Rica

\section{Prácticas inclusivas centradas en el aprendizaje: un estudio de casos múltiples en educación infantil}

López Jiménez, Tatiana; Castillo Venegas, Catalina; Taruman Monsalve, Javiera; Urzúa Calderón, Allison Prácticas inclusivas centradas en el aprendizaje: un estudio de casos múltiples en educación infantil

Revista Educación, vol. 45, núm. 1, 2021

Universidad de Costa Rica, Costa Rica

Disponible en: http://www.redalyc.org/articulo.oa?id=44064134014

DOl: https://doi.org/10.15517/revedu.v45i1.40536

\section{(c) $(1) \Theta$}

Esta obra está bajo una Licencia Creative Commons Atribución-NoComercial-SinDerivar 3.0 Internacional. 


\title{
Prácticas inclusivas centradas en el aprendizaje: un estudio de casos múltiples en educación infantil
}

\author{
Inclusive Learning Practices: A Multiple Case Study in Early Childhood Education
}

Tatiana López Jiménez

Pontificia Universidad Católica de Valparaiso, Chile tatiana.lopez@pucv.cl

(iD http://orcid.org/0000-0002-5870-6853

Catalina Castillo Venegas

Pontificia Universidad Católica de Valparaiso, Chile

castillovenegascatalina@gmail.com

(iD http://orcid.org/0000-0003-0853-9943

\section{Javiera Taruman Monsalve}

Pontificia Universidad Católica de Valparaiso, Chile

javierataruman@gmail.com

(iD http://orcid.org/000-0002-6534-5531

\section{Allison Urzúa Calderón}

Pontificia Universidad Católica de Valparaíso, Chile

madeurzua@hotmail.es

(iD http://orcid.org/0000-0002-9959-3867,
DOI: https://doi.org/10.15517/revedu.v45i1.40536

Redalyc: http://www.redalyc.org/articulo.oa?id=44064134014

\section{Resumen:}

Este estudio se enmarca en el contexto chileno, donde la inclusión en educación infantil se ha incorporado gradualmente en las políticas públicas mediante decretos y orientaciones curriculares que velan por el desafío de avanzar en el derecho de una educación más igualitaria, que elimine toda forma de discriminación, tanto de acceso al aprendizaje, como del desarrollo de todos los educandos. El objetivo del estudio es caracterizar prácticas inclusivas centradas en el aprendizaje que desarrollan maestras de educación infantil en el nivel transición ( 4 a 6 años). Para ello se utilizó una metodología cualitativa con enfoque interpretativo, basado en un estudio de casos múltiples. Participaron tres maestras de educación infantil, a quienes se les aplicó una entrevista semiestructurada y una observación participante en 18 situaciones de aprendizaje. Los datos se analizaron mediante un análisis de contenido y una interpretación descriptiva de las observaciones del aula. Los resultados señalan que las maestras implementan prácticas inclusivas en las que favorecen, en mayor medida, las dimensiones organización del juego y el aprendizaje, y movilización de recursos en el aula. Por otra parte, se identifica con menor presencia la dimensión diversificación de la enseñanza. Se discuten las escasas modalidades empleadas por las maestras en su práctica pedagógica para favorecer la representación, la expresión y la motivación por el aprendizaje, principios esenciales para el desarrollo del aprendizaje. Además, se revela la importancia que presenta la experiencia de aprendizaje para la adquisición y retroalimentación de conceptos y conocimientos oportunos para las niñas y los niños de primera infancia.

Palabras ClaVe: Diversificación de la educación, Educación infantil, Inclusión, Práctica pedagógica, Proceso de aprendizaje.

\section{Abstract:}

Children's education in the Chilean context has gradually become a part of public policies through decrees and curricular guidelines that safeguard the right to more egalitarian education, eliminating all types of discrimination that impact student access to learning and development. This study aims to describe inclusive learning practices developed by early education teachers for transitional levels (ages 4 to 6). A qualitative methodology was used based on a multiple case study and an interpretative approach. Three early childhood education teachers participated in a semi-structured interview involving 18 classroom scenarios. The data 
was then examined through content analysis and descriptive interpretations based on classroom observation. The results obtained reveal that teachers use inclusive practices that largely favor organizing play and learning as well as resource mobilization in the classroom. However, classroom diversification was lacking with few modalities among teachers to favor representation, expression and motivation towards learning, essential principles of learning development. The study also reveals the importance of learning for the timely acquisition of knowledge and feedback of concepts in early childhood students.

KEYwORDS: Diversified Education, Early Childhood Education, Inclusion, Learning Process, Pedagogical Practice.

\section{INTRODUCCIÓN}

La literatura da cuenta de la importancia que ha tenido la educación infantil en las agendas públicas de diversos países (Barrero, 2016; Morago\#n-Alcan\#iz y Martínez-Bello, 2016), este auge se explica por la abundante evidencia científica y multidisciplinaria que ha mostrado impactos positivos, tanto individuales como sociales en la educación de la primera infancia (Grande y González, 2015). El objetivo del estudio es caracterizar las prácticas inclusivas orientadas al desarrollo del aprendizaje de la niñez, en que cada docente ajusta sus actuaciones pedagógicas para responder a su óptimo desarrollo.

La motivación de estudiar las prácticas inclusivas centradas en el aprendizaje en educación infantil, se sustenta en el interés de indagar en cómo las maestras y los maestros implementan prácticas pedagógicas oportunas para responder al desarrollo y aprendizaje de niñas y niños, y conocer el grado en que las acciones pedagógicas que declaran ejecutar, responden a las políticas de inclusión propuestas para este nivel educativo. Políticas que expresan la importancia de una enseñanza de calidad fundada en los valores y principios de los Derechos del Niño (Manghi, Saavedra y Bascuñan, 2018; Ministerio de Educación de Chile [MINEDUC], 2018; Ministerio de Educación, 2015).

De acuerdo con Booth, Ainscow y Kingston (2006); Booth y Ainscow (2015), la inclusión educativa es un enfoque que responde a la diversidad de las personas y a las diferencias individuales, entendiendo que la diversidad es una oportunidad para el enriquecimiento de la sociedad. Por su parte, Echeita y Ainscow (2011) destacan que la práctica de la inclusión requiere de un conjunto de acciones relevantes, entre ellas identificar y eliminar barreras que impidan el ejercicio de una educación inclusiva, también, responder a aquel estudiantado que se encuentre con mayor riesgo de vulnerabilidad, ya sea por marginación, exclusión o fracaso escolar.

En los últimos años, la literatura revela diversos estudios interesados en la inclusión y sus implicancias para la educación (Ainscow, 2012; González-Gil, Martín-Pastor y Poy, 2019; Manghi et al., 2018; Muñoz, 2018), a pesar de que sus objetos de estudio y diseño metodológico varían, coinciden en destacar la importancia del maestro, sus competencias y creencias sobre la diversidad, como una condición fundamental para el éxito de la inclusión educativa.

En cuanto a la inclusión en educación infantil los estudios expresan que esta se ha implementado de manera paulatina, pero con importantes hitos, se destaca la Declaración de Salamanca del año 1994 y el Índex para la inclusión infantil (Booth et al., 2006). La evidencia empírica muestra que hay un mayor avance en términos de cultura y política (González-Gil et al., 2019), pero que la práctica es todavía una tarea pendiente de consolidarse (Balongo y Mérida, 2016). Los resultados de estudios enfocados en educación infantil señalan que las maestras y los maestros elaboran adaptaciones del proceso de enseñanza (Barrero, 2016), sin embargo, estas adaptaciones ocasionalmente favorecen el ambiente para el aprendizaje de todos los educandos, dado que solo se orientan a nivel de diseño del proceso de enseñanza y aprendizaje (Grande y González, 2015). $\mathrm{O}$ bien, se enfocan mayoritariamente en las necesidades educativas especiales (NEE) derivadas o no de discapacidad (Figueroa, Gutiérrez y Velásquez, 2017).

De acuerdo con Muntaner, Rossello y De la Iglesia (2016) y Chadwell, Roberts y Daro (2019), se requiere de una formación de maestros más especializada para que otorguen una respuesta educativa diversificada en el aula, la cual les permita no centrarse únicamente en niños con discapacidad, sino que se encarguen de 
reducir las barreras hacia el aprendizaje de toda la niñez. Esto implica, tener en cuenta la diversidad como un aspecto propio del aula, que ofrezca a las niñas y los niños apoyos especializados continuos, utilice diferentes formas para que expresen y representen su aprendizaje, sus motivaciones y necesidades (Barrero, 2016), lo que permitiría de parte de cada docente una mayor atención a la diversidad, más participativa y de calidad (Grande y González, 2015).

Para el desarrollo del objetivo planteado en este estudio, se presentan a continuación los antecedentes teóricos y prácticos referidos a la inclusión en educación infantil, luego la metodología adoptada y, posteriormente, los resultados, discusiones y conclusiones del estudio.

\section{Antecedentes teóricos y prácticos referidos a la inclusión en educación infantil}

Entre los desafíos que presenta la educación se encuentra la preocupación por responder a una sociedad caracterizada por la diversidad (Ainscow, 2001; González-Gil et al., 2019). Este reconocimiento adquiere especial relevancia en la etapa de educación infantil, dada su creciente importancia en el desarrollo de las habilidades cognitivas, psicosociales, y la configuración de identidad (Barrero, 2016). Este primer nivel educativo presenta principios pedagógicos como la singularidad, potencialidad (Mineduc, 2018) que favorecen una educación que responde a las diferencias propias de cada educando (Solís, Pedrosa, y Mateos, 2019), que puede concretarse en una variedad de metodologías activas- participativas, que ofrece a los educandos diversas oportunidades para que puedan alcanzar su máximo desarrollo (Balongo y Mérida, 2016).

La literatura muestra que la inclusión en educación infantil, se concreta mediante tres dimensiones propuestas en el Índex de educación infantil de Booth et al. (2006), crear culturas, elaborar políticas y desarrollar prácticas inclusivas que resaltan la importancia que tiene el juego, el aprendizaje en la infancia, así como la colaboración con otros en un contexto de aceptación y valoración, en el cual participan niñas y niños, sus familias y profesionales del centro escolar.

En este estudio la práctica pedagógica es entendida como espacios de actividad conjunta en constante cambio y movimiento, interdependientes entre sí, y caracterizadas por un lenguaje, un espacio-tiempo, y relaciones sociales particulares, más o menos distintivas, comunes y compartidas (Mauri, Clarà, Ginesta y Colomina, 2013; López, 2017). Esta forma de comprender la práctica pedagógica, asume que un conjunto de acciones seleccionadas e intencionadas llevadas a cabo por las maestras y los maestros, y basado en los elementos del currículo, tendrá cierto grado de implicación en los aprendizajes de los infantes.

La relevancia de implementar una educación infantil inclusiva radica en conocer las características y habilidades de los educandos, para identificar sus avances y retrocesos, así como también las oportunidades que el contexto le puede brindar para su óptimo desarrollo, al permitirle participar en instancias educativas (Echeita y Ainscow, 2011). De acuerdo con Vygotsky (1979), se reconoce la labor mediadora de las maestras y los maestros para promover el aprendizaje de los educandos, quienes a partir de diferentes metodologías brindan un acceso equitativo y participación a las niñas y los niños, para que desarrollen sus habilidades, independientemente de sus características físicas, psíquicas o sociales.

Las maestras y los maestros organizan su trabajo pedagógico a partir de marcos orientadores que explicitan objetivos, condiciones y facilitadores para el proceso de inclusión educativa (Echeita y Ainscow, 2011). Los tres marcos considerados en este estudio, corresponden al Índex para la inclusión de educación infantil (Booth et al., 2006), el modelo de enseñanza que facilita la puesta en práctica de una enseñanza diversificada, denominado Diseño Universal de Aprendizaje (DUA), finalmente, dado el contexto en que se desarrolla el estudio, se utiliza el Documento orientador para el desarrollo de prácticas Inclusivas en educación parvularia (Subsecretaría de Educación Parvularia, 2018).

Respecto al Índex, su objetivo es generar comunidades escolares colaborativas que fomenten a todo el estudiantado a altos niveles de logro. Mientras que el DUA plantea principios que permiten que todo el alumnado pueda acceder al currículum, para así eliminar las barreras de tu entorno y respetar la diversidad 
existente en el aula, estos son las múltiples formas de representación, expresión e implicación (Alba, Sánchez y Zubillaga, 2014). Por su parte, el documento orientador contribuye a la reflexión en diversas materias que impactan en la vida de las niños y los niños, y apunta a generar condiciones de equidad de oportunidades (Subsecretaría de Educación Parvularia, 2018).

Con la óptima implementación de los marcos orientadores se pretende que las maestras y los maestros disminuyan barreras que dificulten una educación para todos, lo cual da mayor importancia al rol activo de las niñas y los niños en su aprendizaje, y el juego como mediador de aprendizaje. Tal como lo señalan las dimensiones propuestas en el índex para la inclusión, cultura, políticas y práctica (Booth et al., 2006), que trabajan de manera interconectadas en pro del óptimo funcionamiento de una educación inclusiva. El personal docente ajusta sus acciones pedagógicas en el aula a partir de una propuesta de planificación centrada en atender a la diversidad, adapta los contenidos, objetivos y actividades, centrándose en las condiciones y oportunidades que ofrecerá a los educandos para que aprendan y se desarrollen, e incorpora equipamientos heterogéneos y apoyos necesarios para garantizar la igualdad de oportunidades de todo el alumnado sin excepciones.

Este tránsito desde una práctica pedagógica hacia una que propenda la inclusión en el aula, requiere necesariamente una mirada sistémica del proceso educativo, así como de colaboración entre distintos profesionales, que respondan a altos niveles de participación por parte del estudiantado (Gelber, Treviño, González, Escribano y Ortega, 2019). La toma de decisiones que el cuerpo docente ejecuta en el aula, es esencial para eliminar las dificultades que enfrenta el estudiantado para aprender e insertarse cabalmente en la comunidad educativa (Booth y Ainscow, 2015).

Finalmente, es relevante señalar que de los últimos estudios desarrollados en el contexto chileno referidos a la educación inclusiva, destacan aquellos orientados a las percepciones de los educadores frente a estudiantes migrantes (Aravena, Riquelme, Mellado, Villagra, 2019), las estrategias curriculares desarrolladas por los técnicos en educación infantil para una educación inclusiva en un jardín infantil (Muñoz, 2018); además, otros estudios ponen de manifiesto que la diversificación de la enseñanza es un desafío y oportunidad de aprendizaje profesional docente (Chadwell et al., 2019), puesto que dicha diversificación está escasamente presente en el aula (San Martín, Salas, Howard y Blanco, 2017).

Así, el objetivo general de este estudio es caracterizar las prácticas inclusivas que propicien el desarrollo y aprendizaje de las niñas y los niños (5 a 6 años), a partir del cual se plantean dos objetivos específicos: el primero, indagar en cómo las maestras declaran implementar los aspectos curriculares que consideran relevantes para promover la inclusión y propiciar el aprendizaje de la niñez del nivel; y el segundo, conocer las acciones pedagógicas inclusivas que las maestras efectúan en el aula para favorecer el aprendizaje de todos sus educandos.

\section{Metodología}

\section{Diseño}

Con el fin de contribuir a una descripción y análisis que permitan caracterizar las prácticas pedagógicas a partir de los datos y en triangulación con el marco referencial referido a la inclusión centrada en el aprendizaje, se optó por una metodología cualitativa, con enfoque interpretativo (Bisquerra, 2012) y un diseño de casos múltiples (Stake, 2010), en que se estudia el discurso y la implementación de la práctica pedagógica de maestras de educación infantil en su contexto natural. 


\section{Participantes}

La elección de los casos tiene un carácter teórico, basado en la pertinencia con la temática en estudio, se seleccionaron por criterio de expertos, con la finalidad de contar con maestras que permitan comprender el fenómeno a investigar (Stake, 2010). Dada la variedad de centros educativos existentes en el contexto nacional ${ }^{[1]}$. Se establecieron tres criterios de selección, a saber: que las maestras pertenezcan a instituciones educativas de diferente dependencia; que la institución educativa cuente con programa de Inclusión Escolar [2] (PIE) o Educadoras Diferenciales ejerciendo; las maestras cuenten con más de 15 años de experiencia laboral y deseen participar voluntariamente en el estudio.

La caracterización de la muestra se presenta en la Tabla 1, en cada caso se señala: dependencia del centro educativo, años de experiencia y grado de formación. Las maestras se desempeñan en el nivel de transición, con un promedio de 30 educandos de entre 4 a 6 años.

TABLA 1

Descripción de las participantes

\begin{tabular}{ll|l|l}
\hline Casos & Dependencia & Años de Experiencia & Grado académico \\
\hline Caso 1 & Municipal & 36 años & Licenciada en Educación \\
Caso 2 & Particular pagado & 20 años & M(c) educación inicial \\
\hline Caso 3 & Particular pagado & 20 años & Licenciada en Educación \\
\hline
\end{tabular}

Fuente: Elaboración propia

Fuente: Elaboración propia

\section{Instrumentos de recogida de datos}

El estudio se desarrolló mediante la entrevista semiestructurada y observación participativa (Flick, 2007). El objetivo de la entrevista fue acercarse al punto de vista subjetivo de las participantes, comprendiendo mediante una conversación las acciones que desarrollan en el aula, al igual que sus condiciones y la implicancia con el aprendizaje de todos los educandos. Esta se diseñó a partir de tres dimensiones teóricamente informadas propuestas en el índex para la inclusión de educación infantil (Booth et al., 2006) y el documento orientador de las prácticas inclusivas (Subsecretaría de Educación Parvularia, 2018). Además, la entrevista previa a su aplicación fue validada mediante juicio de expertos, las dimensiones son:

- $\quad 1^{a}$ Dimensión Organizando el juego y el aprendizaje: refiere a los aspectos de la planificación que la maestra considera para responder al aprendizaje, la diversidad y estrategias para promover la participación de todos las y los niños. Implica, conocimientos previos, conformación de grupos, apoyos otorgados y evaluación de progresos (Booth et al., 2006).

- $2^{a}$ Dimensión Movilización de recursos: involucra por parte de la maestra la selección e implementación de materiales que promueven la inclusión de las niñas y los niños. Además, implica recursos humanos y materiales, distribución y utilización de estos y el trabajo colaborativo entre los párvulos (Booth et al., 2006).

- $\quad 3^{a}$ Dimensión Diversificación de la enseñanza: refiere a las propuestas que elabora la maestra para el aprendizaje de todos los educandos, basado en la consideración de experiencias que enriquecen las formas de percepción de la información, creando respuestas por medio de diversas modalidades de 
apoyo y de comprometer a los educandos con la participación en su aprendizaje (Subsecretaría de Educación Parvularia, 2018).

En cuanto a la observación participativa, su objetivo fue identificar prácticas pedagógicas inclusivas que propicien el aprendizaje de todos los niños y las niñas del nivel. Se efectuaron diferentes observaciones del escenario natural y cotidiano en que las maestras implementan las experiencias de aprendizajes en el aula (Flick, 2007). Dichas observaciones se registraron en una pauta de observación, diseñada a partir del análisis inductivo (Mayring, 2014) realizado a las respuestas obtenidas por las entrevistas de las participantes. La pauta se organizó de acuerdo con las dimensiones propuestas en la entrevista, se propusieron categorías e indicadores observables y previo a su utilización se ejecutó el proceso de validación interna (Mayring, 2014).

Luego de que cada participante firmara un consentimiento informado, se procedió al proceso de recogida de datos, planificado para un semestre académico (marzo - julio, 2019). Las entrevistas se dieron al inicio de la investigación, su duración aproximada fue de 40 minutos. Las observaciones participativas del aula se calendarizaron en conjunto con cada maestra durante un mes y medio. Para cada caso se concretaron seis observaciones de 45 minutos aproximadamente, en las que se visualizan una situación de aprendizaje orientada a ámbitos de aprendizaje propuestos en el currículo para el nivel de educación infantil (MINEDUC, 2018). En total se analizaron tres entrevistas y 18 situaciones de aprendizajes que corresponden a los tres casos examinados.

\section{Procedimiento de análisis de datos}

En las entrevistas semiestructuradas se desarrolló un análisis de contenido (Mayring, 2014), el cual implicó previamente transcribir la información mediante una enumeración por líneas. La unidad de análisis fue el segmento del mensaje (Mayring, 2014), y las categorías se identificaron de manera inductiva.

A las entrevistas se les elaboraron tres niveles de codificación, la primera codificación abierta, con la finalidad de crear las categorías basadas en las unidades de análisis. Este proceso se ejecutó de forma individual y luego en pareja, con la finalidad de identificar las discrepancias y registrarlas para el control de la fiabilidad. La segunda codificación, correspondió a una codificación selectiva, se describió e interpretó el significado de cada una de las categorías identificadas. La tercera codificación, corresponde a una codificación axial, en la cual compararon y unificaron las categorías vinculándolas por conexiones conceptuales. Esta última etapa se organizó de manera grupal por todas las autoras del estudio, con el fin de aunar temáticas expresadas en cada caso, definirlas, y relacionarlas con las dimensiones previamente establecidas. El control de la fiabilidad del análisis de la entrevista se efectuó mediante un procedimiento de acuerdo inter-jueces, basado en la concordancia consensuada (Mayring, 2014). Las discrepancias en la codificación de las categorías se resolvieron entre las jueces, se discutieron los resultados hasta alcanzar un acuerdo y refinar las categorías. El proceso finalizó una vez alcanzada la saturación de categorías.

En cuanto a las pautas de observación, estas fueron generadas de forma inductiva a partir de las respuestas de las participantes obtenidas mediante la entrevista. En las observaciones del aula, las investigadoras adoptaron un papel de observador activo (Flick, 2007), donde no se involucró con los participantes, más bien, se mantuvo un rol de observador.

En la pauta de observación, la unidad de análisis fue la actuación pedagógica de la maestra en su práctica. Una vez finalizadas las seis observaciones planificadas para cada caso (en total 18 observaciones), se elaboró un cuadro resumen por cada caso, con la finalidad de identificar las prácticas que la maestra desarrolla con mayor y menor presencia en su contexto natural cuando implementa el proceso de enseñanza y aprendizaje en el aula. A fin de identificar aspectos característicos de cada uno de los casos y comprender sus particularidades, se ejecutó una sumatoria de las prácticas observadas, se obtuvieron los puntajes totales por indicador y por categoría, con el fin de identificar una regularidad entre las categorías propuestas en cada dimensión. 
Finalmente, se identificó la presencia de la dimensión en la práctica de la maestra, considerando el puntaje obtenido en relación con el puntaje máximo de la categoría. En la Tabla 2 se ejemplifica el procedimiento desarrollado en un caso y en la dimensión organizando el juego y el aprendizaje.

TABLA 2

Ejemplo análisis pauta de observación a partir de las dimensiones y categorías Caso 1

\begin{tabular}{|c|c|c|c|c|c|c|c|c|}
\hline Dimensión & Organ & o el jue & el apr & aje & & & & \\
\hline Categoría & Monit & del apre & aje & & & & & \\
\hline Indicadores & Día 1 & Día 2 & Día 3 & Día 4 & Día 5 & Día 6 & $\begin{array}{l}\text { Puntaje } \\
\text { obtenido }\end{array}$ & $\begin{array}{l}\text { Puntaje } \\
\text { máximo }\end{array}$ \\
\hline $\begin{array}{l}\text { Constata que el párvulo } \\
\text { comprenda las instrucciones } \\
\text { Existe seguimiento sobre el } \\
\text { progreso de los párvulos }\end{array}$ & 5 & 4 & 3 & K4 & 5 & 4 & 25 & 30 \\
\hline durante el desarrollo de la & 1 & 5 & 3 & 4 & 5 & 4 & 22 & 30 \\
\hline $\begin{array}{l}\text { experiencia de aprendizaje. } \\
\text { Verifica que el párvulo } \\
\text { desarrolle las actividades } \\
\text { propuestas. }\end{array}$ & 55 & 54 & 53 & 55 & 55 & 55 & $\begin{array}{c}27 \quad 74 \\
82,2 \%\end{array}$ & 3090 \\
\hline
\end{tabular}

Fuente: Elaboración propia

\section{Resultados}

Se presentan los resultados obtenidos del análisis de los tres casos objeto de estudio, a partir de los cuales se busca caracterizar prácticas inclusivas orientadas al desarrollo y el aprendizaje de las niñas y los niños. Se inicia con los resultados de las tres entrevistas semiestructuradas, seguido de los resultados de la pauta de observación participativa, aplicada a las 18 situaciones de aprendizajes observadas.

El primer núcleo de resultados, corresponde al análisis de contenido efectuado a las entrevistas, permitió responder al primer objetivo específico, indagar en cómo las maestras declaran implementar los aspectos curriculares que consideran relevante para promover la inclusión y propiciar el aprendizaje de todos los niños y las niñas del nivel. Los resultados muestran un total de trece categorías organizadas y contenidas en las tres dimensiones propuestas. Cada categoría permite comprender cómo las maestras garantizan la participación y aprendizaje de todos los niños y las niñas en las situaciones de aprendizaje que ejecutan en el aula. En la Tabla 3 se detallan la organización de las categorías por cada una de las dimensiones. 
TABLA 3

Dimensiones y sus categorías

\begin{tabular}{|c|c|c|c|}
\hline Dimensiones & $\begin{array}{l}\text { Organizando el juego y el } \\
\text { aprendizaje }\end{array}$ & Movilización de recursos & $\begin{array}{l}\text { Diversificación de la } \\
\text { enseñanza }\end{array}$ \\
\hline Categorías & $\begin{array}{l}\text { 1. Estrategias de enseñanza 2. Diseño } \\
\text { de la planificación } 3 \text {. Juego y } \\
\text { aprendizaje } 4 \text {.Trabajo colaborativo } \\
\text { entre párvulos } 5 \text {. Manejo efectivo del } \\
\text { aula } 6 \text {. Monitoreo del aprendizaje } 7 . \\
\text { Instrumentos evaluativos } 8 \text {. } \\
\text { Promoción del aprendizaje } \\
\text { participativo }\end{array}$ & $\begin{array}{l}\text { 1. Funcionamiento del equipo } \\
\text { educativo. } 2 \text {. Recursos } \\
\text { pedagógicos. } 3 \text {. Participación de la } \\
\text { familia en el aprendizaje. }\end{array}$ & $\begin{array}{l}\text { 1.Implementación de } \\
\text { la planificación } \\
\text { 2.Múltiples formas de } \\
\text { enseñanza. }\end{array}$ \\
\hline
\end{tabular}

Fuente: Elaboración propia

La organización de las categorías en las tres dimensiones varía desde ocho para la primera, y dos en la última dimensión, evidenciando que las maestras enfatizan en una situación de aprendizaje en la organización del juego por sobre la diversificación de la enseñanza. A continuación, por cada dimensión se presenta una definición operacional de las categorías, seguido de un ejemplo discursivo de las maestras que se enfatiza en aquellas que tuvieron mayor y menor reiteración en el conjunto de casos analizados.

\section{$1^{a}$ Dimensión Organizando el juego y el aprendizaje}

Se identificaron ocho categorías asociadas. La primera alude a estrategias de enseñanza, se define como las acciones que organiza la maestra mediante la gestión de diversos apoyos, los cuales promueven la participación y aprendizaje de los párvulos, considerando las necesidades educativas especiales y agrupamiento dentro del aula (MINEDUC, 2018).

La segunda, diseño de la planificación, incluye los momentos en que las participantes refieren al proceso previo a la implementación, el momento en que se organiza el proceso de enseñanza y aprendizaje, considerando los contextos específicos y las condiciones y particularidades del estudiantado. (MINEDUC, 2018).

La tercera categoría, juego y aprendizaje, refiere aquellos fragmentos del discurso en que las maestras dan cuenta del principio pedagógico del marco orientador de educación infantil, el juego es considerado como un mediador del aprendizaje, e impulsor del desarrollo de las niñas y los niños. Es así como puede ser una actividad natural por parte del párvulo, como también una estrategia pedagógica por parte de la maestra (MINEDUC, 2018).

La cuarta categoría, trabajo colaborativo entre párvulos, se define como las acciones que desarrolla la maestra para otorgar posibilidades de interacciones entre los párvulos en variadas actividades y juegos de carácter colaborativo (MINEDUC, 2018).

La quinta categoría, manejo efectivo del aula, refiere a los momentos en que la maestra alude al clima en el que se implementa el proceso de enseñanza y aprendizaje, caracterizado por el respeto, la relación entre estudiantes, la promoción de relaciones positivas y otros elementos que propicien un buen ambiente escolar (MINEDUC, 2018).

La sexta categoría, monitoreo del aprendizaje, se define como el proceso en que la educadora desarrolla un seguimiento de la comprensión y apropiación del contenido por parte de los párvulos, y utiliza estrategias pertinentes para evidenciar el progreso en sus aprendizajes (Booth y Ainscow, 2015). 
En tanto, la séptima categoría instrumentos evaluativos, refiere a las herramientas pedagógicas que utilizan las maestras como parte del proceso educativo para registrar y evidenciar de manera sistemática el progreso del aprendizaje de los párvulos. Se espera que, a partir de los resultados obtenidos, la maestra retroalimente el aprendizaje y la práctica pedagógica (MINEDUC, 2018).

En cuanto a la octava categoría, promoción del aprendizaje participativo criterio fundamental en el desarrollo de las prácticas inclusivas, implica que la docente en su discurso va integrando activamente a las niñas y a los niños en el juego y aprendizaje, promoviendo sus interacciones y participación (Booth y Ainscow, 2015).

Respecto a la ejemplificación desde el discurso de las participantes, los resultados muestran que la categoría con mayor reiteración en los tres casos objeto de estudio, corresponde a estrategias de enseñanza. Se ejemplifica a continuación:

(...) al finalizar, ocupamos muchas preguntas para que el niño se dé cuenta de cómo aprendió, o sea, que lo hagamos pensar, preguntas más desafiantes para que ellos realicen como una meta cognición, o sea, 'en qué te tuviste que fijar', 'cómo llegaste', 'cómo lo hiciste', entonces, en el fondo ellos tienen que verbalizar todo lo que realizaron tanto en matemática, en lenguaje, en lo que sea. (Entrevista Caso 3, comunicación personal, 19 de diciembre 2018).

Mientras que la categoría que presentó menor reiteración en el discurso de las participantes, en los tres casos objeto de estudio, se encuentra la referida a la promoción del aprendizaje participativo, en la cual la maestra indica que:

(...) a través de la metodología de la pregunta que es su\#per importante, poder saber a través de eso que está pensando el niño, como va, y que también, lo verbalice, que verbalice su acción porque a medida que el niño más lo diga, más consciente se va a hacer, de alguna manera. (Entrevista Caso 2, comunicación personal, 14 de diciembre 2018).

\section{2a Dimensión Movilización de recursos}

En esta dimensión se identifican tres categorías. La primera, funcionamiento del equipo educativo, refiere a las acciones en que la maestra alude las responsabilidades profesionales que presenta en el aula, específicamente el diálogo sobre aspectos pedagógicos y didácticos entre maestras, u otros educadores del centro educativo, que apoyan la labor pedagógica al interior del aula ( MINEDUC , 2008).

La segunda categoría, recursos pedagógicos, comprende el uso de materiales educativos y de apoyo al proceso de enseñanza y aprendizaje que son utilizados durante el desarrollo de situaciones de aprendizaje que se espera, resulten ser significativos para los párvulos, y que, por ende, puedan otorgarles un sentido a partir de sus conocimientos previos, experiencias e intereses ( MINEDUC, 2008).

En cuanto a la tercera categoría, participación de la familia en el aprendizaje, esta se define como las formas y modos que emplea la maestra para involucrar y dar cuenta a las familias del proceso de aprendizaje de sus hijos, es fundamental integrar a cuidadores para que se sientan bienvenidos, respetados y escuchados (MINEDUC, 2018).

Respecto a la ejemplificación desde el discurso de las participantes, la categoría con mayor reiteración corresponde a funcionamiento del equipo educativo, la cual se ejemplifica en el siguiente extracto: “(...) tiene que ser algo conciso y preciso, y en relación con eso, que me focalizó con mi compañera en los grupos que requieren mayor atención, mayor apoyo, para poder realizar el desafío que hemos propuesto". (Entrevista Caso 2, comunicación personal, 14 de diciembre 2018).

Por otra parte, la categoría con menor reiteración en el discurso de las participantes corresponde a participación de la familia en el aprendizaje, donde la maestra señala:

(...) Bueno, aquí se realiza reunión de apoderados tres veces en el semestre. Les muestro las carpetas, con sus trabajos, con los avances que han tenido en general, porque yo no tomo casos particulares en reuniones y, todas las semanas, o sea cada dos 
semanas cuando voy cambiando de unidad, voy informando a los apoderados vía agenda, para que ellos puedan en sus casas también apoyarlos en lo que nosotros estamos haciendo. (Entrevista Caso 1, comunicación personal, 10 de diciembre 2018).

\section{$3^{a}$ Dimensión Diversificación de la enseñanza}

En cuanto a la tercera dimensión se encuentran dos categorías. La primera, múltiples formas de enseñanza, refiere en primer lugar a las acciones en que las maestras otorgan múltiples medios para representar la información, ajustándose a la diversidad del estudiantado y sus diferentes modos de representar y comprender el mundo que les rodea. En segundo lugar, el proporcionar diversas posibilidades de expresión de los conocimientos de los educandos y de llevar a cabo las tareas de aprendizaje, ya sea en ocasiones por preferencias o por condiciones personales (Alba et al., 2014).

En lo que respecta a implementación de la planificación, se comprende como aquel momento en que la maestra estructura las situaciones de aprendizaje considerando los saberes, intereses y experiencias de sus estudiantes. Se espera que dicho momento presente una estructura definida que permita diferenciar sus distintas etapas (inicio, desarrollo y cierre), independientemente del tipo de estrategia desarrollada (MINEDUC, 2018).

Respecto a la ejemplificación desde el discurso, se presenta con mayor reiteración en el discurso de las maestras la categoría implementación de la planificación, la maestra señala:

(...) la clase tiene tres etapas, inicio, desarrollo y cierre. En el inicio, uno presenta lo que quiere, yo no pongo el objetivo, porque mis alumnos algunos no leen, pero ahí en el inicio tú empiezas a indagar los conocimientos previos... Entonces ya, ahí va la motivación. “A ver, ¿qué les parece si realizamos esto?” Luego empiezas a desarrollar tu clase, dependiendo del objetivo que yo quiera lograr en ese momento, es el material que se presenta. (Entrevista Caso 1, comunicación personal, 10 de diciembre 2018).

Por el contrario, se presenta con menor reiteración la categoría múltiples formas de enseñanza, ejemplificándose a continuación:

(...) tratamos de que todos los niños puedan aprender a través de los distintos estilos, entonces la misma experiencia de aprendizaje involucra diversas estrategias y metodologías como para que el niño que tiene problemas y el que no, logre aprender todas las metas que tenemos. Entonces, se trabaja con mucho material visual, se trabaja con PPT, con video, con YouTube. (Entrevista Caso 3, comunicación personal, 19 de diciembre 2018).

En lo que respecta al segundo núcleo de resultados referidos al análisis de la pauta de observación aplicada en los tres casos y en las 18 situaciones de aprendizaje, este permitió responder al segundo objetivo específico, conocer las acciones pedagógicas inclusivas que las maestras ejecutan en el aula para favorecer el aprendizaje de todos sus educandos.

Dado la extensión de este núcleo de resultados, se presentan únicamente aquellas categorías identificadas con mayor regularidad e irregularidad en el quehacer pedagógico de las maestras. Presentando así resultados globales, de los tres casos analizados; se efectuó un ajuste en las categorías, prescindiendo de la categoría diseño de la planificación y participación de la familia en el aprendizaje, por su dificultad de observación en la implementación del proceso de enseñanza.

En cuanto a la primera dimensión, organizando el juego y el aprendizaje, los resultados señalan que, en los tres casos, la categoría monitoreo del aprendizaje posee mayor regularidad, presentándose en la mayoría de las situaciones de aprendizajes observadas en el aula; las maestras de educación infantil verifican constantemente que las niños y los niños desarrollen las actividades propuestas y comprendan las instrucciones. Además, llevan un seguimiento sobre el progreso de cada uno durante el desarrollo de la situación de aprendizaje. Por el contrario, con mayor irregularidad se encuentra la categoría de instrumentos evaluativos, lo que evidencia en que en la mayoría de las situaciones de aprendizaje observadas no se explicitan los instrumentos de evaluación; 
la maestra del caso 2 emplea diversos insumos para dar cuenta del progreso de sus educandos. Sin embargo, en los casos 1 y 3, los resultados indican que las maestras hacen uso de un solo tipo de herramienta de evaluación.

Respecto a la segunda dimensión, movilización de recursos, los resultados señalan que, en todos los casos, la categoría que presenta mayor regularidad corresponde al uso de los recursos pedagógicos, las observaciones desarrolladas a las diferentes situaciones de aprendizaje en el aula permitieron identificar que, los recursos se utilizan como apoyo al proceso de enseñanza y aprendizaje, además estos son de buena calidad, llamativos y contextualizados a las niñas y los niños. Por el contrario, la categoría con mayor irregularidad es funcionamiento del equipo educativo, debido a que en los casos 2 y 3 hubo predominancia en las decisiones tomadas en conjunto, a diferencia del caso 1 , quien no ejecutaba un trabajo colaborativo con su asistente de sala.

Finalmente, en la tercera dimensión, diversificación de la enseñanza, se observa con mayor regularidad la categoría implementación de la planificación. Es relevante destacar que, en todos los casos, en la mayoría de las situaciones de aprendizaje existe una baja presencia de los criterios observados, pues se evidencia la ausencia de los tres momentos de la clase, en particular el promover un cierre metacognitivo al culminar las sesiones. Por el contrario, la categoría que mayor irregularidad es múltiples formas de enseñanza, puesto que los casos 2 y 3 consideraron dos o más medios para entregar la información, como también diversos medios para que el párvulo exprese su aprendizaje, a diferencia del caso 1, quien consideró una única forma en las distintas observaciones elaboradas durante el proceso.

\section{Discusión}

El objetivo del estudio se concretó en caracterizar prácticas inclusivas centradas en el aprendizaje que desarrollan maestras de educación infantil. Participaron tres casos objeto de estudio, a los cuales se elaboraron entrevistas semiestructuras y seis observaciones participativas del aula, para cada caso. Tanto las entrevistas, como las observaciones del aula, se organizaron a partir de tres dimensiones teóricamente informadas sustentadas en los marcos orientadores para la inclusión, Índex para la inclusión infantil y documento orientador de prácticas inclusivas; ambos responden a las normativas, decretos y orientaciones legales que tienen el poder performativo de regular las prácticas pedagógicas del aula.

Los resultados presentados ponen de relieve la importancia de estudiar las prácticas inclusivas centradas en el aprendizaje, a partir situaciones de aprendizajes que se implementan en su contexto natural. La relevancia de las tres dimensiones: organizar el juego y el aprendizaje, movilizar los recursos y diversificar la enseñanza, radica en que estas han sido ampliamente difundidas en políticas educativas que propenden a la inclusión (Ainscow, 2012; Barrero, 2016; Booth y Ainscow, 2015). Además, este estudio, permitió operacionalizar dichas dimensiones en categorías observables, a fin de poder caracterizar aquellas actuaciones prácticas que organizan las maestras en el aula para propiciar el aprendizaje de todos los educandos.

Las 13 categorías propuestas permiten ampliar los resultados de estudios previos de Grande y González (2015) y Figueroa et al. (2017), puesto que los autores desarrollan un análisis documental y los resultados de este estudio aportan datos empíricos que se sustentan de lo que las maestras declaran implementar en el aula y las observaciones directas que se ejecutan a partir del contexto real en que se estudian los procesos naturales de enseñanza y aprendizaje en educación infantil.

Para caracterizar las prácticas inclusivas centradas en el aprendizaje, los resultados muestran, en primer lugar, que las categorías que presentaron mayor predominio en lo declarado por las maestras corresponden a las estrategias de aprendizaje en la primera dimensión; funcionamiento del equipo educativo en la segunda dimensión e implementación de la planificación en la tercera dimensión. Por su parte, en las observaciones del aula, las categorías que se presentaron con mayor regularidad fueron: monitoreo del aprendizaje, correspondiente a la primera dimensión; recursos pedagógicos de la segunda dimensión e implementación de la planificación de la tercera dimensión. De esta manera, se destaca la importancia que tiene para las maestras 
estructurar las situaciones de aprendizaje considerando los saberes, intereses y experiencias del estudiantado (MINEDUC, 2008).

Por el contrario, los resultados muestran que las categorías que presentaron menor presencia en lo declarado por las maestras corresponden a promoción del aprendizaje participativo en la primera dimensión; participación de la familia en el aprendizaje perteneciente a la segunda dimensión y múltiples formas de enseñanza de la tercera dimensión. A su vez, en las observaciones del aula, las categorías que se presentaron mayor irregularidad corresponden a instrumentos evaluativos, referida a la primera dimensión; funcionamiento del equipo educativo, perteneciente a la segunda dimensión y múltiples formas de enseñanza de la tercera dimensión.

Dichos resultados denotan que tanto lo declarado por la maestras como las observaciones efectuadas en el aula, la categoría múltiples formas de enseñanza correspondiente a la dimensión diversificación de la enseñanza, presenta menor presencia, este hallazgo es coherente con lo propuesto por Figueroa et al. (2017), puesto que destaca la necesidad de presentar a las y los niños de educación infantil la información de variadas formas, para que expresen sus aprendizajes, favoreciendo así su implicación. Este hecho, de acuerdo con Grande y González, (2015) es crucial y relevante para favorecer el óptimo aprendizaje de las niñas y los niños de educación infantil. Por otra parte, la categoría que refiere a la baja participación que presentan las niñas y los niños en su aprendizaje, es coherente con lo señalado por García y López-Pastor (2015), pues estos deben ser el centro del proceso de enseñanza, y las maestras y los maestros considerar su forma de aprender, implementando estrategias que permitan utilizar todos sus sentidos mediante experiencias enriquecedoras. Además, Morago\#n-Alcan\#iz y Martínez-Bello, (2016) ponen de relevancia la falta de apertura de oportunidades para el juego y la expresión en el aula de educación infantil, aspecto fundamental para plantear distintas posibilidades para que los educandos puedan expresar lo que sienten y comprenden.

Por otra parte, la baja presencia, de la categoría referida a instrumentos de evaluación, es coherente con investigaciones precedentes (García y López-Pastor, 2015), que señala que las maestras y los maestros de educación infantil otorgan retroalimentación a los educandos incentivando a que a aprendan de los errores y aciertos, reconduciendo el proceso de enseñanza y aprendizaje; sin embargo, estas escasamente se sistematizan para fines evaluativos sumativos, pues no se elabora un registro sistematizado de los aciertos y avances de los educandos.

Es así, que los resultados obtenidos en el análisis de los casos dan cuenta de una disociación entre el discurso declarado en las entrevistas ejecutadas a las maestras y el quehacer pedagógico implementado en el aula, ya que, en las entrevistas, las maestras destacan la importancia del funcionamiento educativo, propuesta en la dimensión movilización de recursos, sin embargo, en las observaciones del aula, la misma categoría tiene una escaza o nula presencia. Este hecho pone de relieve lo señalado por González-Gil et al. (2019), quien destaca la necesidad de que las maestras desarrollen una mayor reflexión en torno al quehacer pedagógico llevado a cabo en las aulas de educación infantil.

Los resultados presentados, van en la línea de lo planteado por Solís et al. (2019), quienes establecen que este nuevo paradigma inclusivo ha actualizado el rol del profesorado, no obstante, estos cambios no han ido precedidos de un análisis de sus creencias, actitudes y cómo estas se relacionan con las acciones y prácticas profesionales. Este hallazgo se ve reflejado en los resultados, puesto que muestran que en las prácticas educativas de las maestras, la dimensión que presenta menos categorías es la referida a la diversificación de la enseñanza, evidenciando así su baja presencia en las observaciones del aula, lo que lleva a concluir la importancia de repensar, desde las nuevas políticas públicas, el quehacer en el aula (Gelber et al., 2019) e indagar en la actitud de las maestras hacia la inclusión (González-Gil et al., 2019). 


\section{Conclusiones}

Los resultados de este estudio permiten concluir que la educación inclusiva no está\# promoviendo un cambio de paradigma en la educación infantil actual, más bien se encuentra de acuerdo con Gelber et al. (2019), en un tránsito a un paradigma inclusivo, que tiene más bien un carácter práctico, asociado a una necesidad educativa especial (NEE). En las prácticas pedagógicas estudiadas, dos de tres de las maestras (caso 1 y 2), tienden a efectuar lo señalado por Barrero (2016), puesto que no incluyen en sus prácticas apoyos especializados homogeneizando así las características de las y los niños, mientras que en el caso 3, la maestra presenta de forma regular en sus prácticas las categorías referidas al monitoreo del aprendizaje y la utilización de recursos variados.

Los resultados presentados deben comprenderse en el contexto que son estudiados, por lo cual no son generalizables, sin embargo, presentan reflexiones interesantes para el desarrollo de la inclusión en el contexto de educación infantil en chile. Si bien se evidencia que en el contexto nacional hay un progreso en las políticas públicas, estas no orientan las prácticas educativas llevadas a cabo por maestras, se podría interpretar que las políticas chilenas se encuentran desarrolladas hacia un extremo integracionista, ignorando así las oportunidades complementarias del ámbito educativo para lograr que exista efectivamente un beneficio y apoyo a todos los educandos. Cabe destacar, que las leyes y decretos implementados en los últimos años en pos de la inclusión, no se han trabajado con las maestras para que exista una real adherencia a ellos, por lo cual, dentro de las aulas se presentan dificultades para respuesta a la diversidad, lo que se evidencia en la baja presencia de las categorías correspondientes a la dimensión orientada a la diversificación de la enseñanza.

A modo de cierre, resulta imperioso incorporar la atención a la diversidad desde la formación inicial y continua de las maestras infantiles. Una visión clara, acompañada de estrategias y herramientas, que permitan desarrollar una representación argumentativa (cognitiva o subjetiva) de mayor complejidad, para implementar prácticas pedagógicas, que respondan al aprendizaje de todos los educandos, y no solo aquellos que puedan presentar una necesidad educativa. Considerando que la dimensión más descendida es la diversificación de la enseñanza, es necesario promover un trabajo más colaborativo entre las maestras de educación infantil y el equipo psicopedagógico de los centros escolares. La evidencia indica que las maestras implementan prácticas que se centran en el aprendizaje, pero no cuentan el suficiente conocimiento conceptual y pedagógico para expresar cómo llevar a cabo la inclusión. Como proyecciones para futuros estudios, sería interesante ampliar la muestra del estudio y considerar como parte del objetivo variables que permitan enriquecer los resultados aquí mencionados, como el tipo de establecimiento, los años de experiencia y nivel de estudio y especialización de las maestras.

\section{ReFERENCIAS BibLiográficAS}

Ainscow, M. (2001). Comprendiendo el desarrollo de escuelas inclusivas. Notas y referencias bibliográficas. Recuperado de https://bit.ly/3jaMXss

Ainscow, M. (2012). Haciendo que las escuelas sean más inclusivas: lecciones a partir del análisis de la investigación internacional. Revista Educación Inclusiva, 5(1), 39-49. Recuperado de http://www.ujaen.es/revista/rei/linked /documentos/documentos/15-4.pdf

Alba, C., Sánchez, M., y Zubillaga, A. (2014). Diseño universal para el aprendizaje (DUA). Pauta para su introducción en el curriculo. Madrid: Moreta

Aravena, O., Riquelme, P., Mellado, M. A., y Villagra, C. (2019). Inclusión de Estudiantes Migrantes en la Región de La Araucanía, Chile: Representaciones desde los Directivos Escolares. Revista latinoamericana de educación inclusiva, 13 (1), 55-71. doi: https://doi.org/10.4067/S0718-73782019000100055 
Balongo, E., y Mérida, R., (2016). El clima de aula en los proyectos de trabajo Crear ambientes de aprendizaje para incluir la diversidad infantil. Perfiles Educativos 38(152), 146-162. Recuperado de http://www.scielo.org.mx/p df/peredu/v38n152/0185-2698-peredu-38-152-00146.pdf

Barrero, A. (2016). Lo visible e invisible de la diversidad en la educación infantil. Infancias imágenes, 15(2), 262-270. doi: https://doi.org/10.14483/udistrital.jour.infimg.2016.2.a07

Bisquerra, R. (coord.) (2012). Metodología de la investigación educativa. Madrid: La Muralla.

Booth, T., Ainscow, M., y Kingston, D. (2006). Índex para la inclusión. Desarrollo del juego, el aprendizaje y la participación en Educación Infantil. Salamanca: INICO.

Booth, T., y Ainscow, M. (2015). Guía para la Educación Inclusiva. Desarrollando el Aprendizaje y la participación en los Centros Escolares. Madrid: OEI.

Chadwell, R., Roberts, A., y Daro, A., (2019). Ready to Teach All Children? Unpacking Early Childhood Educators' Feelings of Preparedness for Working with Children with Disabilities. Early Education and Development, 31, 100-112. doi: https://doi.org/10.1080/10409289.2019.1621584

Echeita, G., y Ainscow, M. (2011). La Educación inclusiva como derecho. Marco de referencia y pautas de acción para el desarrollo de una revolución pendiente. Tejuelo, 12, 26-46. Recuperado de https://mascvuex.unex.es/revist as/index.php/tejuelo/article/view/2497

Figueroa, X., Gutiérrez de Pin\#eres, C., y Velázquez, J. (2017). Estrategias de inclusión en contextos escolares. Diversitas: Perspectivas en Psicología, 13(1), 13-26. Recuperado de http://www.redalyc.org/pdf/679/67952833 001.pdf

Flick, U. (2007). Introducción a la investigación Cualitativa (2a Ed.). Madrid: Moreta.

García, S., y López-Pastor, V. M. (2015). Evaluación Formativa y Compartida en Educación Infantil. Revisión de una Experiencia Didáctica. Qualitative Research in Education, 4(3), 269-298. doi: https://doi.org/10.17583/qre.2 015.1269

Gelber, D., Treviño, E., González, A., Escribano R. y Ortega, L. (2019). Del dicho al hecho: creencias y prácticas inclusivas en establecimientos y aulas escolares en Santiago. Perspectiva Educacional, 58(3), 73-101. Recuperado de https://scielo.conicyt.cl/pdf/perseduc/v58n3/0718-9729-perseduc-58-03-73.pdf

González-Gil, F., Martín-Pastor, E. y Poy, R. (2019). Educación inclusiva: barreras y facilitadores para su desarrollo. Análisis de la percepción del profesorado. Profesorado. Revista de Curri\#culum y Formación de Profesorado, 23(1), 243- 263. doi: https://doi.org/10.30827/profesorado.v23i1.9153

Grande, P., y González, M.M. (2015). La educación inclusiva en la educación infantil: propuestas basadas en la evidencia. Tendencias Pedagógicas, (26), 145-162. Recuperado de https://dialnet.unirioja.es/servlet/articulo? $\underline{\text { codigo }=5247176}$

López, T. (2017). Ayuda educativa y reflexión conjunta sobre la propia práctica en el prácticum de educación infantil. (Tesis de Doctorado). Universidad de Barcelona, Barcelona-España.

Manghi, D., Saavedra, C., y Bascuñan, N. (2018). Prácticas Educativas en Contextos de Educación Pública, Inclusión Más Allá de las Contradicciones. Revista Latinoamericana de Educación Inclusiva, 12(2), 21-39.doi: https://do i.org/10.4067/S0718-73782018000200021

Mauri, T., Clarà, M., Ginesta, A., y Colomina, R. (2013). La contribución al aprendizaje en el lugar de trabajo de los equipos docentes universitarios. Un estudio exploratorio. Infancia y Aprendizaje, 36(3), 341-360. doi: https:// doi.org/10.1174/021037013807533025

Mayring, P. (2014). Qualitative content analysis: theoretical foundation, basic procedures and software solution. Klagenfurt, Austria: GESIS. Recuperado de https://nbn-resolving.org/urn:nbn:de:0168-ssoar-395173

Ministerio de Educación de Chile [MINEDUC] (2018). Bases curriculares de educación parvularia. Santiago, Chile: Gobierno de Chile, Ministerio de Educación. Recuperado de https://bit.ly/2Tgitdu

Ministerio de educación. ( 8 de junio, 2015). Ley $\mathrm{N}^{\circ}$ 20.485: Inclusión escolar que regula la admisión de los y las estudiantes, elimina el financiamiento compartido y prohíbe el lucro en establecimientos educacionales que reciben aportes del estado. Diario Oficial de la República de Chile. Recuperado de http://bcn.cl/1uvlu 
Morago\#n-Alcan\#iz, F., y Martínez-Bello, V. (2016). Juegos de niñas y juegos de niños: Estudio sobre la representación del juego infantil a través del dibujo. Revista Educación, 40(1), 1-17. doi: https://doi.org/10.15517/revedu.v4 0 il 17439

Muntaner, J., Rosselló, M., y De la Iglesia, B. (2016). Buenas prácticas en educación inclusiva. Revista Educatio Siglo XXI, 34(1), 31-50. doi: https://doi.org/10.6018/j/252521

Muñoz, F. (2018). Educación Inclusiva: una mirada a las estrategias desarrolladas por las técnicos en Educacio\#n Parvularia de un Jardín Infantil. Polypho\#ni\#a. Revista de Educacio\#n Inclusiva, 2(1), 75-97. Recuperado de ht tps://revista.celei.cl/index.php/PREI/article/view/75-97/pdf

San Martín, C., Salas, N., Howard, S., y Blanco, P. (2017). Acceso al Curri\#tculum Nacional para Todos: Oportunidades y Desafi\#os de los Procesos de Diversificacio\#n de la Ensen\#anza en Escuelas Diferenciales Chilenas. Revista Latinoamericana de Educacio\#n Inclusiva, 11(2), 181-198.doi: https://doi.org/10.4067/S07 18-73782017000200012

Solís, P., Pedrosa, I., y Mateos, L.M. (2019). Assessment and interpretation of teachers' attitudes towards students with disabilities. Cultura y Educación, 31(3), 576-608. doi: https://doi.org/10.1080/11356405.2019.1630955

Stake, R. (2010). Investigación con estudio de casos. Madrid: Morata.

Subsecretaría de Educación Parvularia. (2018). Documento orientador para el desarrollo de prácticas inclusivas en educación parvularia. Santiago, Chile: Gobierno de Chile, Ministerio de Educación. Recuperado de https://bi t.ly/380S9tc

Vygotsky, L. (1979). El desarrollo de los procesos psicológicos superiores. Barcelona, España: Grijalbo.

\section{Notas}

[1] En Chile los establecimientos educacionales reconocidos oficialmente por el Estado pueden ser clasificados, según la naturaleza de su dependencia administrativa y financiera, en municipales, Particulares Subvencionados y particulares pagados.

[2] Programa de Integración Escolar, que rige a partir de la Ley de Inclusión 20.845 PIE se implementa en los establecimientos educacionales regulares, su propósito es favorecer la participación y el logro de los objetivos de aprendizaje de todo el estudiantado, aportando recursos y equiparando las oportunidades educativas especialmente para quienes presentan mayores necesidades de apoyo para progresar en sus aprendizajes. 